\title{
A Review of Hematological Malignancy in Pregnancy
}

\author{
Noor Asikin Mohd Sakri, Zalina Nusee, Ahmad Muzamir Ahmad Mustafa, and Roziah Husin
}

\section{ABSTRACT}

\begin{abstract}
Hematological malignancy in pregnancy is a rare condition which leads to lack of prospective study and randomized control trial. Nevertheless, it has own challenge to the medical field in term of managing patient with the said condition. The dilemma is to decide among the necessities of continuation of pregnancy, the choice of diagnostic tools and chemotherapeutic drugs, and the timing of initiation treatment without disregarding the women's wish and preferences. The process often has a profound psychological burden on patients and family members. Furthermore, delays in diagnosis and intervention will adversely affect the outcomes of pregnancy and the disease itself. The effect of teratogenicity of chemotherapeutic drugs on the fetus and the progression of the disease during pregnancy are the main concern in treating this group of patients. This article will review the management and outcomes of 6 cases of hematological malignancy in pregnancy in one center ( 3 Hodgkin lymphoma, 1 chronic myeloid leukemia, 1 hairy cell leukemia, and 1 myeloproliferative neoplasms). The outcomes of the pregnancy cases in this article were five successful live births with one case of early neonatal death due to prematurity with a history of secondtrimester loss. The treatment was initiated during the second trimester to reduce the risk of chemotherapy to the fetus. Even though a few cases had fetal growth compromise but the fetal outcomes seem to be good with early interventions and multidisciplinary approached.
\end{abstract}

Keywords: Leukemia, lymphoma, myeloproliferative neoplasm, pregnancy.

\section{INTRODUCTION}

Cancer in general and in particular hematological cancer during pregnancy is uncommon. The management is challenging not just for the patients, but also for the medical professionals since involving two lives. Treatment should be balanced in treating the disease depending on the type and stage of cancer with some adjustment to reduce potential adverse effects on the fetal development and the pregnancy itself. A multidisciplinary team shall be involved in managing the said condition for a better holistic approach [1], [2].

In 2019, 6 patients were diagnosed with hematological malignancy during pregnancy. Five patients were newly diagnosed in this current pregnancy, one patient has already been diagnosed in the previous pregnancy in 2015. There were three patients with Hodgkin lymphoma (HL) and one patient each for chronic myeloid leukemia (CML), hairy cell leukemia (HCL), and myeloproliferative neoplasms (MPNs). This report aims to review the management and the effect of treatment on pregnancy and neonatal outcomes. The details of patient presentation, histology, imaging for staging, progress, and outcomes of pregnancy were reviewed via the clinics.

\section{A. Case 1}

Submitted : June 01, 2021

Published : June 23, 2021

ISSN: 2593-8339

DOI: $10.24018 /$ ejmed.2021.3.3.904

Noor Asikin Mohd Sakri*

Department of Obstetrics \& Gynaecology, International Islamic University Malaysia, Kuantan, Pahang, Malaysia.

(e-mail: sikinsakri@ gmail.com)

Zalina Nusee

Department of Obstetrics \&

Gynaecology, International Islamic University Malaysia, Kuantan, Pahang, Malaysia.

(e-mail: drzalina@iium.edu.my)

Ahmad Muzamir Ahmad Mustafa Department of Obstetrics \& Gynaecology, Hospital Tengku Ampuan Afzan, Kuantan, Pahang, Malaysia.

(e-mail: ahmad.muzamir@moh.gov.my) Roziah Husin

Department of Obstetrics \& Gynaecology, Hospital Tengku Ampuan Afzan, Kuantan, Pahang, Malaysia.

(e-mail: roziahhusin74@gmail.com)

*Corresponding Author

patient's medical record from hematology and obstetrics

\section{CASE REPORT}

N.S.I, a 28-year-old Malay lady Gravida 4 Para 3 at 30 weeks of gestation presented with bilateral neck swellings to hematology outpatient clinic. The swellings were rapidly increased in size caused discomfort to the patient. The patient has no systemic B symptoms such as unexplained fever, significant weight loss, and drenching night sweats. A physical examination revealed multiple cervical lymphadenopathies with the gravid uterus at 30 weeks of gestation. No inguinal lymphadenopathy and other examinations were unremarkable.

A cervical lymph biopsy was done under local anesthesia with a sterile technique. The result revealed histologic subtypes of classical nodular sclerosing Hodgkin's Lymphoma (HL). Urgent contrast-enhanced computed tomography (CECT) of neck and thorax reported that the 
patient has extensive bilateral cervical, supraclavicular lymph nodes, and anterior mediastinal matted lymph nodes. The staging was stage II, Bulky disease with primary cervical. The routine blood investigation and echocardiogram revealed normal findings. The patient underwent chemotherapy with doxorubicin, bleomycin, vinblastine, and dacarbazine (ABVD) regime planned for 6 cycles.

Maternal-fetal medicine (MFM) service was obtained for fetal surveillance and co-managed. A multidisciplinary team (MDT) meeting was discussed the timing of delivery to reduce the risk of chemotherapy to the fetus. A monthly transabdominal scan revealed normal fetal growth and Doppler velocimetry. The patient underwent planned induction of labor at 36 weeks of gestation after 14 days completed second cycles of chemotherapy. Healthy baby born with good Apgar score (8/9) and birth weight of 2400 gram. The low molecular weight heparin (LMWH) was started for venous thromboembolism prophylaxis (VTE) prior to diagnosis and continued 6 weeks post-partum. The chemotherapy resumed post-partum and the patient was advised not to breastfeed her baby.

\section{B. Case 2}

N.H.I, a 27-year-old Malay lady Gravida 3 Para $1+1$ at 16 weeks of gestation with a history of second-trimester loss and one previous caesarean section presented with bilateral neck swelling for 5 months duration. The swellings were increasing in size rapidly. She reported has B symptoms such as unexplained loss of weight, intermittent fever, night sweating but no significant compressive symptom and hoarsens of voice. A physical examination revealed bilateral cervical lymphadenopathy, with size $10 \times 6 \mathrm{~cm}$ on right and $3.5 \times 7 \mathrm{~cm}$ on the left side. Abdomen examination noted uterus palpable at 16 weeks gestation and no inguinal lymphadenopathy. The other examinations were unremarkable.

The admission blood investigation showed a hemoglobin (Hb) level of $10.3 \mathrm{~g} / \mathrm{dL}$, white blood cell (WBC) count of $11.06 \times 10^{9} / \mathrm{L}$, lymphocytes count of $1.28 \times 10^{9} / \mathrm{L}$, and platelet (PLT) count of $377 \times 10^{9} / \mathrm{L}$. Serum lactate dehydrogenase (LDH) was elevated. The echocardiogram revealed an ejection fraction of $66 \%$ with normal left ventricular function. A right cervical lymph node biopsy was done under sterile technique. The result revealed histologic subtype of lymphocyte-rich classical HL with the presence of Hodgkin and Reed-Sternberg-like cells with an immunohistochemical pattern positive for CD15 and CD30, negative for panCK, CD20 and EMA. CD3+ cells were seen resetting around the Reed-Sternberg cells. The Ki67 proliferative index was around $60 \%$.

The patient was diagnosed with lymphocyte-rich classical HL with clinical stage III Bx, Bulky disease with primary cervical. The referral to the MFM unit for co-managed and fetal surveillance was made prior to chemotherapy. The multidisciplinary meeting involved the MFM unit and hematological team with the presence of family members were discussed regarding choices of treatment, chemotherapy adverse effect on fetus, pregnancy outcomes, and timing of delivery. The initial trans-abdomen scan revealed a healthy singleton fetus with a parameter around 18 to 19 weeks of gestation. Subsequently, fetal surveillance was done by clinical and ultrasonography. An anatomical scan at 22 weeks gestation revealed no structural anomalies. Fetal growth and Doppler velocimetry were monitored closely every 4 weeks.

The patient underwent chemotherapy ABVD regime started at 17 weeks of pregnancy and planned for 6 cycles. An anticoagulant LMWH was started for VTE prophylaxis prior to diagnosis and continued until 6 weeks post-partum. The initial planned was to induced patient at the 36 weeks of gestation. Unfortunately, the patient delivered prematurely at 28 weeks gestation via spontaneous vertex delivery after completed three cycles of the ABVD regime. A baby weighing 1000 grams was born with a poor Apgar score and needs resuscitation. The baby succumbed on day three of life due to complication of prematurity.

\section{Case 3}

N.A.A, a 27-year-old Malay lady Para 1, who was diagnosed with classical nodular sclerosing HL, stage II, Bulky disease with primary mediastinal at 32 weeks of pregnancy. Initially presented with abdominal pain and was treated as pyelonephritis, clinical examination revealed multiple cervical lymphadenopathies. The diagnosis was confirmed with a cervical lymph node biopsy. Other blood investigations were unremarkable.

The patient underwent chemotherapy with the ABVD regimen soon after diagnosis was made. Consultation with MFM service was made and MDT meetings were arranged. The fetal assessment revealed a normal growing fetus with low amniotic fluid index and pregnancy was induced at 36 weeks of gestation after receiving 1 cycle of ABVD. The patient had an emergency caesarean section due to pathological cardiotocography. The baby was born with a good Apgar score (8/9) with a birth weight of 2700 grams. An anticoagulant LMWH was started for VTE prophylaxis prior to diagnosis and continued until 6 weeks post-partum.

\section{Case 4}

F.N, a 30-year-old Malay lady, Gravida 2 Para 1 at 9 weeks of gestation with underlying chronic myeloid leukemia (CML) presented to hematology outpatient clinic for routine follow-up. The pregnancy was allowed because of disease remained in chronic phase. An oral Imatinib, a tyrosine kinase inhibitor (TKI) was discontinued.

The patient was diagnosed with CML in 2015 during her first pregnancy. Presented with raised WBC count during antenatal blood test which was $192.96 \times 10^{9} / \mathrm{L}$, low Hb level of $8.4 \mathrm{~g} / \mathrm{dL}$, and normal PLT count of $304 \times 10^{9} / \mathrm{L}$ during that time. The bone marrow aspirate and trephine biopsy (BMAT) procedure revealed blasts cells of $2.0 \%$ and reported as a chronic phase. A subcutaneous non-pegylated Interferon Alpha-2b (IFN- $\alpha$ ) 90 mg weekly was initiated during pregnancy and shifted to oral Imatinib $400 \mathrm{mg}$ daily postdelivery. The pregnancy was brought to term and the patient had an uneventful normal vaginal delivery.

The BCR-ABL 1 was monitored every 6 months and the latest level $0.007 \%$ before the current pregnancy. The patient was not on treatment till had molecular relapsed with BCRABL 1 increased to $0.1285 \%$ at 24 weeks gestation, oral Imatinib $400 \mathrm{mg}$ daily reinitiated. IFN- $\alpha$ was not available at that moment. As a result of poor compliance due to worries of side effects to the fetus, BCR-ABL 1 level remained static with loss of complete hematologic response (CHR). The 
WBC count was $14.3 \times 10^{9} / \mathrm{L}$. The treatment was shifted to pegylated IFN- $\alpha 90 \mathrm{mg}$ weekly at 32 weeks of gestation, weekly WBC count had been monitored and continued to show a progressively declined and normalized until delivery.

Consultation from the MFM service was obtained for fetal surveillance and co-managed. An anatomical scan at 20 weeks of gestation revealed no congenital structural anomalies. The serial fetal growth ultrasonography and Doppler velocimetry were normal. Spontaneous labor occurred at 38 weeks of gestation; a healthy baby born with a good Apgar score (8/9) with a birth weight of 2690 grams through normal vaginal delivery. The anticoagulant LMWH was initiated for 3 weeks post-partum for VTE prophylaxis and etonogestrel implant $68 \mathrm{mg}$ (NEXPLANON®) was inserted before discharged home. A follow-up review at 4 weeks post-partum, white cell count level increased to $22.3 \times 10^{9} / \mathrm{L}$, an oral Imatinib $400 \mathrm{mg}$ daily resumed.

\section{E. Case 5}

N.A.I, a 31-year-old Malay lady, Gravida 2 Para 1 at 10 weeks of gestation was referred to a hematology outpatient clinic for pancytopenia on routine blood investigation during first antenatal booking. Significant anemic symptoms included dyspnea, dizziness, and reduce effort tolerance with unexplained fever. Physical examination revealed mild pallor with hepatosplenomegaly and no lymphadenopathy. The $\mathrm{Hb}$ level of $8.4 \mathrm{~g} / \mathrm{dL}$ with no iron deficiency features, WBC count of $3.5 \times 10^{9} / \mathrm{L}$, and PLT count of $44 \times 10^{9} / \mathrm{L}$. A full blood picture test was confirmed true pancytopenia with the presence of occasional suspicious cells. The BMAT procedure confirmed a diagnosis of hairy cell leukemia (HCL) at 14 weeks of gestation.

Referral to MFM service was arranged for co-managed and fetal surveillance. The MDT meeting involved a hematologist and maternal-fetal medicine specialist with patient and spouse emphasized regarding termination of pregnancy, choice of treatment (Cladribine, Rituximab, pegylated IFN$\alpha$ ) and its effect on fetus development, and complication of disease toward pregnancy outcomes itself. The patient and spouse opted for continuation of pregnancy and choose to be treated with subcutaneous pegylated INF- $\alpha 90 \mathrm{mg}$ weekly which is a less adverse effect on the fetus. The treatment commenced at 15 weeks and was planned until 36 weeks of gestation.

At the start of the treatment, a weekly complete blood count showed a decrease in the trend of $\mathrm{Hb}, \mathrm{WBC}, \mathrm{PLT}$, and absolute neutrophil count with required blood transfusion. The size of splenomegaly was not changed. However, towards the end of pregnancy, a normal complete blood count was presented which shows that the patient was positively responded to the pegylated INF- $\alpha$ treatment. Throughout the pregnancy, the patient has no bleeding complications and easy fatigue was her only symptom.

A two weekly trans-abdominal ultrasound revealed small gestational age (SGA) fetus growth with normal Doppler velocimetry. The patient was induced at 37 weeks of gestation and had a normal vaginal delivery with no postpartum hemorrhage with her platelet count remains normal $\left(170 \times 10^{9} / \mathrm{L}\right)$. A baby born with a good Apgar score (8/9) and birth weight of 2490 grams with a normal platelet count at birth. Post-partum LMWH was initiated for 3 weeks as VTE prophylaxis. A follow-up review showed a decreased trend of plate count, hence at five months, post-partum chemotherapy Cladribine was initiated. She has inserted an intra-uterine contraceptive device at the pre-pregnancy clinic.

\section{F. Case 6}

N.B.I, a 33-year-old Malay lady Gravida 1 Para 0 at 17 weeks of gestation presented with pan-cytosis on routine blood investigation during ward admission for hyperemesis gravidarum. The $\mathrm{Hb}$ level of $18 \mathrm{~g} / \mathrm{dL}$, hematocrit $57 \%$, WBC count of $20.5 \times 10^{9} / \mathrm{L}$ and PLT count of $774 \times 10^{9} / \mathrm{L}$. A full blood picture test confirmed the findings.

The physical examination and abdomen ultrasound were normal. A positive Janus kinase 2 (JAK-2) V617F mutation test confirmed of diagnosis of myeloproliferative neoplasms (MPNs) with subtype essential thrombocythemia (ET). The patient declined the BMAT procedure. She was followed up regularly and started on acetylsalicylic acid (ASA) $100 \mathrm{mg}$ daily prior to diagnosis and initiated treatment with subcutaneous pegylated IFN- $\alpha 90 \mathrm{mg}$ weekly at 20 weeks of gestation. The treatment was changed to oral hydroxyurea $500 \mathrm{mg}$ daily at 28 weeks because of poor response. Venesection was scheduled as an additional treatment for the patient. LMWH initiated antenatally prior to diagnosis and continued until 6 weeks post-partum for VTE.

The patient was seen in the MFM unit at 21 weeks of gestation for co-managed and fetal surveillance. Fetal growth monitoring and Doppler velocimetry monitoring revealed intrauterine growth restriction (IUGR) with abnormal Doppler velocimetry. A planned caesarean section was scheduled at 30 weeks of gestation and a baby girl was born with a good Apgar (8/9) score with a birth weight of 907 grams. The newborn required resuscitation and was admitted to the Neonatal Intensive Care Unit (NICU) for prematurity and respiratory distress syndrome. A routine complete blood count was normal. The newborn was discharged home after three months of hospital stayed.

The patient was discharged home well post-partum and continued treatment with oral hydroxyurea $500 \mathrm{mg}$ daily and LMWH for 6 weeks post-partum. She was inserted IUCD at the pre-pregnancy clinic. The follow-up PLT count showed raised in trend which was $1039 \times 10^{9} / \mathrm{L}$, therefore oral hydroxyurea was increased to $1000 \mathrm{mg}$ daily together with oral ASA $100 \mathrm{mg}$ daily.

\section{DISCUSSION}

Delayed diagnosis and initiation of the chemotherapeutic drug will influence the prognosis of the disease. A multidisciplinary approach involving obstetricians, hematologists, neonatologists, and radiologist is mandatory to provide the best care of pregnant women with hematological malignancy. An extensive and holistic discussion in regard to treatment options, safety, and efficacy of chemotherapeutic drugs, outcomes of pregnancy, and patient's wishes would need to be addressed and discussed. As far as the treatment option is concerned, many reports suggest avoiding the first trimester for chemotherapy administration. Thus, the treatment should be started after the second trimester onwards to reduce the teratogenicity effect on the fetus. 
The most common hematological malignancy associated with pregnancy are lymphomas in which HL is more frequent than Non-Hodgkin lymphoma (NHL) in pregnant women. A total of $3 \%$ of HL patients are diagnosed during pregnancy [1], [3], [4]. In this article, there were three cases of HL in pregnancy (Case report 1, 2, 3) with two successful live births. One case of early neonatal death due to severe prematurity with a history of second-trimester loss can be one of the contributing factors and not caused by chemotherapy drugs solely. HL in pregnancy has been cited with the low complication of preterm delivery and IUGR [4], [5]. The use of the ABDV regime is safe chemotherapy in pregnancy to be initiated during the second-trimester above. There is no available safety data of the usage of other chemotherapy drugs during pregnancy (e.g., 'Stanford V' or 'BEACOPP') [2]. All patients were diagnosed at the second and third trimesters, therefore early intervention and treatment were initiated to make sure optimal outcomes. Termination of pregnancy should be considered if the diagnosis is made in the early first trimester of gestation such that early chemotherapy can be initiated. Alternatively, the treatment can be delayed to the second trimester if the disease is localized and in the early stage [6], [7].

In this article, there were two patients with leukemia (Case report 4 and 5). Case report 4 with CML in chronic phase diagnosed in the first pregnancy and has achieved major molecular response (MMR) with oral Imatinib, a tyrosine kinase inhibitor (TKI). As a recent practice, treatment with TKIs is the gold standard of care in patients with CML and has been proven will improve the long-term survival rate in most CML patients. The patient was on TKIs for four years before embarked on the current pregnancy. According to previous studies, the patient will have a comparable survival rate to the general population MMR achieved after 2 years of treatment with oral Imatinib [8].

The main issue in this patient is she was on oral Imatinib before pregnancy and only noticed her pregnancy at 9 weeks of gestation. The study showed oral Imatinib or other tyrosine kinase inhibitors (TKI) are at high risk of congenital anomalies [1]. Fortunately, an anatomical scan done at 20 weeks of gestation revealed no congenital structural anomalies. Nevertheless, stopping the oral Imatinib in the first trimester is still debatable. As for the patient, oral Imatinib was discontinued at early of pregnancy causing her disease to progress with molecular relapsed evidence of increased BCR-ABL level $>0.1 \%$ at 24 weeks of gestation. The pegylated IFN- $\alpha$ was commenced on her. IFN- $\alpha$ is a safe drug that can be used throughout gestation with no adverse effect on the fetus [2], [9], [10], [22], [23]. IFN- $\alpha$ inhibits cell proliferation through its effect on protein synthesis, RNA breakdown, and possibly by immunomodulation. It does not cross the placental barrier because of its high molecular weight $(19 \mathrm{kDa})$ and has a protective effect on the fetus [10], [11]. There are two significant case reports discussed regarding the safety profile of IFN- $\alpha$ in pregnancy [24], [25]. The reports elucidated the outcomes of 40 patients including 8 with CML, 27 with ET, two with HCL and carriers of hepatitis $\mathrm{C}$, and one with multiple myeloma and their newborn baby. 8 out of 40 patients were treated with IFN- $\alpha$ in the first trimester and there were no reports of congenital anomalies post-delivery. In this patient, her pregnancy was brought to term with the good achievement of the molecular response.

Case report 5 discussed regarding patient with HCL which generally uncommon B-cell lymphoproliferative neoplasm and it is rare in pregnancy. The information and literature on this condition are limited. This patient presenting with anemic symptoms and splenomegaly. She was treated with a pegylated form of IFN- $\alpha$ with a good response. Her symptom gets improved with the static size of the splenomegaly. There are a few case reports discussed regarding HCL in pregnancy [14]-[17]. The first case report was in 1987 which is limited treatment, and the only choice is post-partum splenectomy [14]. In the recent case reports, successful treatment has been documented with IFN- $\alpha$, biological agent (rituximab), and purine analogs. Laparoscopic splenectomy is one of the established surgical treatments [15]-[17].

Case report 6 is an MPNs with subtype ET. ET is the most common MPNs in childbearing age women and has been studied in pregnancy. However, there is insufficient data being published. Generally, MPNs patients have an increased rate of miscarriage, IUGR, preterm delivery, and intrauterine death with maternal complications occur in about $8 \%$ of patients $[18,19]$. A meta-analysis has reported the outcomes of 461 pregnancies in women diagnosed with ET [20]. The live birth rate was moderate $(50 \%$ to $70 \%$ ) with high firsttrimester loss occurred up to $40 \%$ and late pregnancy losses at $10 \%$. The rate of IUGR $(4.5 \%)$ was higher than in the general population with a high risk of post-partum thrombotic events and post-partum hemorrhage (5.2\%).

In MPNs patient, increased risk of thrombotic events is caused by raised of hematocrit and platelet count and this condition worsen in pregnancy. This risk was reduced starting the second trimester due to increased plasma volume. Nevertheless, hematocrit and platelet levels start to rise again post-partum leading to an increased thrombotic risk. A weekly complete blood count is important during the first six weeks post-partum to detect high-level thrombocytosis or polycythemia recurrent [18]. For this patient, oral ASA and LMWH were initiated antenatally and LMWH was continued to 6 weeks post-partum for VTE prevention. In low-risk patients, single oral ASA can be initiated antenatal and changed to LMWH after delivery [19].

Oral hydroxyurea needs to be avoided in the first trimester due to the high teratogenicity effect. As per the guideline, most studies suggested non-pegylated IFN- $\alpha$ use as a drug of choice in pregnant women with MPNs due to safety profile. Pegylated IFN- $\alpha$ is a pregnancy category $\mathrm{C}$ drug and needs to be used if the benefit outweighs the risk [21]-[23]. Unfortunately, in this case, a subcutaneous pegylated form of INF was used given the availability of the medication with close fetal monitoring. The patient underwent venesection as an additional treatment once at 20 weeks when the platelet counts persistently raised more than $700 \times 10^{9} / \mathrm{L}$. According to the study, there is a role of venesection (cytoreduction) in ET if platelet count raised more than 1000 to $1500 \times 10^{9} / \mathrm{L}$. As a disease complication, the patient underwent idiopathic premature labor due to severe IUGR with evidence of placental insufficiency. 


\section{CONCLUSION}

The outcomes and prognosis of hematological malignancy in pregnant women are comparable with non-pregnant women if treatment is not delayed. Multiple factors are essential to be considered in managing the patients including the staging of the disease, safety of the imaging modalities and chemotherapeutic drugs in terms of teratogenicity effect, the feasibility of treatment, the necessity of continuation of pregnancy, women desire, and expectations. Fortunately, the outcomes of delivery were favorable even chemotherapy started prenatally. Nevertheless, more study is required to be conducted through prospective study and randomized control trial (RCT) for this group of patients focusing on treatment and management for better understanding. Presently, most of the research involving animal studies.

\section{CONFLICT OF INTEREST}

The authors have no conflicts of interest to declare related to this manuscript.

\section{FUNDING}

No funding was received in preparation of this manuscript.

\section{REFERENCES}

[1] Lavi N, Horowitz NA, Brenner B. An Update on the Management of Hematologic Malignancies in Pregnancy. Women's Health. May 2014:255-266.

[2] Mahmoud, Hossam K., Mohamed A. Samra, and Gamal M. Fathy. "Hematologic Malignancies during Pregnancy: A Review." Journal of Advanced Research 1 July 2016: 589-596. Journal of Advanced Research. Web.

[3] Sanchez M, Pellicer B, del Puig Cozar M, Martínez-Sanjuan V, Villegas C, Carbonell F. Hodgkin lymphoma in pregnancy: a case report. Clin Adv Hematol Oncol. 2013;11(8):533-536.

[4] Connors JM. State-of-the-art therapeutics: Hodgkin's lymphoma. J Clin Oncol 2005;23:640-840.

[5] Lishner M, Zemlickis D, Degendorfer P, Panzarella T, Sutcliffe SB, Koren G. Maternal and fetal outcome following Hodgkin's disease in pregnancy. Br J Cancer 1992;65(1):114-7.

[6] Weisz B, Meirow D, Schiff E, Lishner M. Impact and treatment of cancer during pregnancy. Expert Rev Anticancer Ther 2004;4 (5):889902.
[7] Pentheroudakis G, Pavlidis N. Cancer, and pregnancy: poena magna, not anymore. Eur J Cancer 2006;42(2):126-40.

[8] Palani, R., Milojkovic, D. \& Apperley, J.F. Managing pregnancy in chronic myeloid leukemia. Ann Hematol 94, 167-176 (2015).

[9] Pons JC, Lebon P, Frydman R, Delfraissy JF. Pharmacokinetics of interferon-alpha in pregnant women and fetoplacental passage. Fetal Diagn Ther 1995;10(1):7-10.

[10] Baer MR, Ozer H, Foon KA. Interferon-alpha therapy during pregnancy in chronic myelogenous leukemia and hairy cell leukemia. Br J Hematol 1992; 81:167-169.

[11] Mubarak AA, Kakil IR, Awidi A, Al-Homsi U, Fawzi Z, Kelta M, AlHassan A (2002) Normal outcome of pregnancy in chronic myeloid leukemia treated with interferon-a in 1st trimester: report of 3 cases and review of the literature. Am J Hematol 69:115-118.

[12] Shapira T Preg D \& Lishner M How I treat acute and chronic leukemia in pregnancy. Blood Rev 2008;247-259.

[13] Hernández-Ruano K, Morales-Santos D, Portillo-Cálix P, et al. Hairy Cell Leukemia - Case Reports. Med Case Rep. 2016, 2:2.

[14] J. Kell Williams, Hairy cell leukemia in pregnancy: A case report, American Journal of Obstetrics and Gynecology, Volume 156, Issue 1, 1987, Pages 210-211.

[15] Shackleton L, Langabeer SE, O'Brien D, et al. Hairy Cell Leukemia Masquerading as Pancytopenia in Pregnancy. Case Rep Hematol. 2019; 2019:3238168. Published 2019 Aug 21.

[16] Treatment of hairy cell leukemia during pregnancy: are purine analogs and rituximab viable therapeutic options. Daver $N$, Nazha $A$, Kantarjian HM, Haltom R, Ravandi F Clin Lymphoma Myeloma Leuk. 2013 Feb; 13(1):86-9.

[17] Laparoscopic splenectomy for hairy cell leukemia in pregnancy. Adeniji BA, Fallas M, Incerpi M, Hamburg S, Katz R, Ogunyemi D Case Rep Med. 2010.

[18] Barbui T, Finazzi G. Special issues in myeloproliferative neoplasms. Curr Hematol Malig Rep 2011;6(1):28-35.

[19] Harrison CN, Robinson SE. Myeloproliferative disorders in pregnancy. Hematol Oncol Clin North Am 2011;25(2):261-75.

[20] Barbui T, Finazzi G. Myeloproliferative disease in pregnancy and other management issues. Hematology 2006;2006(1):246-52.

[21] McMullin MF, Bareford D, Campbell P, Green AR, Harrison C, Hunt $\mathrm{B}$, et al. Guidelines for the diagnosis, investigation, and management of polycythemia/erythrocytosis. Br J Haematol 2005;130(2):174-95.

[22] Séror J, Sentilhes L, Lefebvre-Lacoeuille C, Marpeau L. Interferonalpha for treatment of essential thrombocythemia during pregnancy: case report and review of the literature. Fetal Diagn Ther 2009;25(1):136-140.

[23] Hiratsuka M, Minakami H, Koshizuka S, Sato I. Administration of interferon-alpha during pregnancy: effects on fetus. J Perinat Med. 2000;28(5):372-376.

[24] Vantroyen B, Vanstraelen D (2002) Management of essential thrombocythemia during pregnancy with aspirin, interferon alpha $2 \mathrm{a}$ and no treatment. A comprehensive analysis of the literature. Acta Hematol 107:158-169.

[25] Mathieu P, Emilie C, Fabrice P, Olivier P. Pregnancy and myeloproliferative neoplasms: A retrospective monocentric cohort. Obstet Med. 2017 Dec; 10(4): 165-169. 